\title{
J osé López Sánchez, miembro de la Generación del 30, comunista, internacionalista e historiador de la medicina
}

\author{
J ose Lopez Sanchez, a member of the 30's Generation, \\ a communist, an internationalist and a medical historian
}

MSc. María del Carmen Amaro Cano

Escuela Nacional de Salud Pública. La Habana, Cuba.

\section{RESUMEN}

J osé López Sánchez (1911-2004). Desde 1925 se incorpora a las actividades revolucionarias, integrándose a la Generación del 30. Militante del Partido Comunista de Cuba, Brigadista Internacional, Comisario Político y Corresponsal de Guerra durante la Guerra Civil española. Historiador de la medicina. Al triunfo revolucionario, en 1961, ocupa el cargo de Viceministro de Salud Pública, logra la reapertura de la Cátedra de Historia de la Medicina y crea el Museo Histórico de las Ciencias "Carlos J. Finlay". Como historiador de las ciencias tiene una amplia bibliografía en la que se destacan sus libros: "Vida y obra del médico habanero Tomás Romay y Chacón" y "Finlay y la verdad científica". Embajador en la India y Suiza. Director de Documentación del Ministerio de Relaciones Exteriores. Su obra en el campo de la investigación histórica de las ciencias fue reconocida con el otorgamiento de la condición de Miembro de Honor de la Unión de Historiadores de Cuba y de la Sociedad Latinoamericana de Historia de la Ciencia y la Tecnología, Investigador de Mérito de la Academia de Ciencias de Cuba, Premio Nacional de Ciencias Sociales 1999 y la Orden de Mérito Científico "Carlos J. Finlay". La Academia también le reconoce su magisterio en la enseñanza de la Historia de la Medicina y le otorga la condición de Profesor de Mérito del Instituto Superior de Ciencias Médicas de La Habana.

Palabras clave: Generación del 30, comunista/brigadista internacional, historiador de la medicina. 


\section{ABSTRACT}

J ose Lopez Sanchez (1911-2004). Since 1925 he participated in revolutionary actions and became a member of the 30's Generation. He was militant of the Cuban Communist party, an international brigade member, political commissioner and war correspondent during the Spanish civil war and also medical historian. At the triumph of the revolution in 1961, he held the post of public health vice-minister, managed to re-open the Medical History department and created "Carlos J . Finlay" historical museum of sciences. As a historian of sciences, he has extensive bibliography of which outstanding books are "Vida y obra del médico habanero Tomás Romay y Chacón" and "Finlay y la verdad cientifica". He was ambassador to India and Switzerland and Director of Documentation at the Ministry of Foreign Affairs. His valuable work in the field of historical research of sciences deserved the award of Honor Member of the Union of Historians of Cuba and of the Latin American Society of Sciences and Technology History, Emeritus Researcher of the Academy of Sciences of Cuba, the National Prize of Social Sciences 1999 and the Order of Scientific Merit "Carlos J. Finlay". The Academy also recognized his teaching work as professor of medical history and he was awarded as Emeritus Professor of the Higher Institute of Medical Sciences of Havana.

Key words: the 1930 's Generation, communist and international brigade member, medical historian.

\section{NTRODUCCI ÓN}

La lucha social estalla en la Universidad de La Habana en 1923 con un movimiento de reforma, liderado por Julio Antonio Mella, quien agrupa a las fuerzas revolucionarias en torno a la ideología marxista y constituye el embrión del que se forman todas las denominadas generaciones. La Generación del 30 -nombre acuñado por Raúl Roa- tuvo como objetivo principal el derrocamiento de la dictadura instaurada por Gerardo Machado. ${ }^{1}$

López Sánchez formó parte de esa generación. Entre sus compañeros de lucha de aquella etapa destacan Rubén Martínez Villena y Pablo de la Torriente Brau, este último, amigo entrañable, casi un hermano. También José Zacarías Tallet, Juan Marinello Vidaurreta y María J osefa Vidaurreta, Pepilla, su inseparable esposa y Raúl Roa García, con quienes sostuvo una profunda amistad fraterna; Carlos Rafael Rodríguez, Nicolás Guillén, Mirtha Aguirre, Emilio Roig de Leuchsering y Antonio Núñez J iménez, camaradas hasta el final de sus días, todos fallecidos antes que él.

Desde 1925, López Sánchez se incorpora a las actividades revolucionarias. Integra la Liga J uvenil Comunista. Participó activamente como miembro de la Comisión Organizadora Pro Libertad de Julio Antonio Mella y, años más tarde, en 1930, en la marcha estudiantil donde fue herido Pablo de la Torriente Brau y asesinado Rafael Trejo. $^{2}$

Contaba apenas 21 años cuando en 1932 se integró al Partido Comunista de Cuba, que apenas contaba con siete años de fundado por Julio Antonio Mella, Carlos Baliño y Alfredo López. Al año siguiente fue Secretario General de la célula del Partido en el Hospital de Emergencias, en momentos en que se fraguaba una 
prórroga de poderes para Machado, prevista para el año siguiente; pero el pueblo estaba hastiado de crímenes e injusticias. Secretamente se preparaba una potente y decisiva huelga general de la que fue mentor y artífice Rubén Martínez Villena y que finalmente logró la caída del tirano el 12 de agosto de ese año.

Pocos días después sobreviene el 4 de septiembre, movimiento promovido por un grupo de sargentos al frente del cual está Fulgencio Batista. El gobierno de Carlos Manuel de Céspedes y Quesada es derrocado y en su lugar se instaura una pentarquía.

Tras la disolución de la Pentarquía asume la presidencia de la República el eminente profesor de Fisiología de la Escuela de Medicina, doctor Ramón Grau San Martín. Estados Unidos se niega a reconocer al nuevo gobierno, que estaba también integrado por Antonio Guiteras. La presencia del destacado luchador en el gabinete le proporciona al nuevo gobierno un matiz popular, especialmente a partir de la intervención de la compañía de electricidad, medida que contó con amplio respaldo del pueblo.

El 29 de septiembre de 1933, una verdadera concentración de pueblo se organiza para dar sepultura a las cenizas de Mella que habían sido traídas desde México por J uan Marinello y Mirta Aguirre. El pueblo le rindió guardia de honor a la urna donde estaban las cenizas del dirigente estudiantil y comunista.

Rubén Martínez Villena, ya bien enfermo, también le rindió guardia de honor. Los asistentes le pidieron que dijera unas palabras. Con voz sonora, pero haciendo pausas a causa de su enfermedad, hizo una corta pero hermosa arenga "Julio Antonio Mella ha regresado dijo para continuar siendo útil a su pueblo y darle sostén a las ideas socialistas. "13

El año 1934 se iniciaba con una acción y propaganda más intensa del Partido Comunista, a partir de la presencia de las cenizas de Mella y la muerte de José Elías Borges Carreras, este último doctor en medicina, cirujano auxiliar del hospital de Emergencias, quien fuera Miembro de los Manicatos, y expulsado de la Universidad en 1927, por lo que marchó a Francia con la intención de continuar sus estudios de medicina; pero de allí fue también expulsado por gestiones del gobierno de Machado; viéndose obligado a viajar a Bélgica donde, finalmente, logró graduarse. Regresó a La Habana y participó en las asambleas depuradoras en la Universidad. ${ }^{4}$

Cuando ocurre el fallecimiento de Gabriel Barceló, el 3 de febrero de ese mismo año 1934, López Sánchez no pudo asistir a su sepelio pues guardaba prisión en El Príncipe, en unión de su compañero de estudios de medicina, Carlos Font Pupo, ambos acusados de subversión y atentado contra el gobierno.

Antonio Guiteras recién había comenzado a organizar un partido, la J oven Cuba, de matiz izquierdista y antiimperialista. El Partido Comunista, interesado en concertar con Guiteras un pacto en relación con la huelga que se venía proyectando, encomienda a López Sánchez - que integraba la comisión del Partido, junto a los doctores Luis Díaz Soto, Luis Alvarez Tabío y el estudiante Carlos Font Pupo, que participaba en los preparativos de la huelga de médicos- la misión de visitarlo.

\section{LA HUELGA DE 1935}

La huelga estalló finalmente en marzo de 1935, fecha luctuosa cuyo desastre fue previsto por Pablo, Guiteras y el Partido Comunista, oportunidad que aprovechó el 
gobierno para limpiar la retaguardia de opositores. El Partido Comunista sufrió la pérdida de numerosos y valiosos cuadros que habían tenido que pasar a la clandestinidad. El terror, el pánico y los despidos masivos de empleados públicos, maestros, alumnos de hospitales y hasta médicos, constituyeron el epílogo de la huelga. La Federación Médica de Cuba fue clausurada.

Luego de aquellos agitados días, Guiteras cree llegado el momento de salir ilegalmente del país con el propósito de armar una expedición para venir a combatir a la Isla. Confió el secreto a Carmelo González, comandante y amigo personal, quien le traicionaría. Batista envió el ejército al sitio por donde se efectuaría la salida con la orden de capturarlo vivo o muerto. Guiteras y Aponte resistieron heroicamente en El Morrillo, lugar cercano a la ciudad de Matanzas, donde finalmente encontraran la muerte.

En esos tiempos el aparato del partido y el movimiento sindical eran atacados sistemáticamente por el gobierno; a lo cual se sumó la existencia de problemas internos en el seno de la organización comunista cubana, ocasionando grandes tensiones políticas. ${ }^{5}$

En 1935 fue designado Secretario General del Comité Seccional de los barrios habaneros de los Sitios y Cayo Hueso y un año después integró el Departamento de Organización del Comité Central del Partido.

Entre tanto, el PRC (Auténtico) se desmembró, aunque la jefatura política se mantuvo en manos del doctor Ramón Grau San Martín; la J oven Cuba, tras la muerte de Guiteras y el encarcelamiento de algunos de sus integrantes, se convirtió en el bocado al que aspiraban algunos de sus más oportunistas miembros. Pablo de la Torriente Brau, desde su exilio en EE. UU., intuyó que era necesario la creación de un nuevo partido con un programa capaz de unir a todos los revolucionarios con un propósito esencial: luchar contra el imperialismo. Así surgió la Organización Revolucionaria Cubana Antimperialista (ORCA). ${ }^{6}$

En palabras de López Sánchez:

Para Pablo el tiempo se mide por las acciones en función de un ideal. Su tesis es clara y precisa: el Frente Único Antimperialista, aunque sabe que todo proceso, y el de la revolución más que ningún otro, requiere de etapas de controversias y sobre todo realismo, sin dejar los sueños que alimentan las utopías.

Pablo afirmaba:

La revolución no es un espejo que refleja siempre toda la imagen. Es un proceso realizado por hombres y, como tal, puede generar un círculo vicioso capaz de crear confusión. Siempre habrá quienes sepan lo que realmente quieren y cómo conducirse mejor. No se pueden sobrestimar las fuerzas y, menos aún, suponer debilidad, idiotez y cretinismo ante los antagonistas.

Finaliza con este magistral apotegma, válido para todos los tiempos: "lo único malo que tiene la revolución está en los revolucionarios". ${ }^{7}$

A pesar de la clausura impuesta a la Universidad, el movimiento estudiantil logra constituir el Comité Estudiantil Universitario (CEU), pequeño embrión del Frente Único, integrado por militantes de diversos partidos. Su principal líder fue Carlos Rafael Rodríguez. 
Blas Roca y Jorge Antonio Vivó se encontraban en Moscú como delegados al VII Congreso de la Internacional Comunista. Al regreso de Blas a La Habana, su principal tarea fue explicar la importancia de crear frentes obreros y populares y se coloca en primer plano la solidaridad internacional con la causa del pueblo español en su defensa de la república.

\section{LA GUERRA CI VI L ESPAÑOLA}

En julio de 1936 estalla la Guerra Civil Española y al año siguiente el Comité Central del Partido Comunista dispuso que López Sánchez fuera el Secretario General de su Comité Seccional en la Universidad de La Habana. Como delegado de la FEU llega ese mismo año a España, para participar en el Congreso Mundial de Estudiantes que se celebraría en Valencia. Allí, como cuadro profesional del Partido Comunista de Cuba, se queda a luchar por la República, integrando la Brigada Internacional, pocos meses después de la muerte de Pablo de la Torriente Brau, su amigo y compañero entrañable.

López Sánchez asume las tareas de comisario político y corresponsal de guerra del periódico "Al Ataque", órgano de la Primera Brigada Móvil de Choque, comandada por Valentín González, El Campesino, y de la que Pablo era comisario político en el momento de la muerte de Pablo en combate en Majadahonda, a escasos doce kilómetros de Madrid.

Entre 1937 y 1938, López Sánchez conoció al General español Enrique Lister y otros dirigentes republicanos, con quienes confraternizó rápidamente. También al comandante Policarpo Martínez Candón, al músico trinitario Julio Cuevas y a otros revolucionarios cubanos, también combatientes que habían ido a España a defender la República.

En palabras propias de López Sánchez:

La guerra revolucionaria de España fue un atrayente foco para la voluntad internacionalista de muchos cubanos. Con entera independencia del Ilamamiento de la Internacional Comunista, los hombres, particularmente los comunistas, fueron por voluntad y medios propios a escalar las montañas nevadas de los Pirineos en busca de los frentes de combates. Desde Cuba, tras soportar los rigores de una lucha perdida, y aún sin reponerse de las heridas dejadas tras la huelga de marzo de 1935, hombres y mujeres marcharon en número casi de mil y en suelo español mostraron ardor, valentía y sacrificio. Pablo de la Torriente Brau es el símbolo más acabado de aquellos internacionalistas. Su vida fue hoguera y fontana. Fue a España a servirla y a aprender para su deber con sus dos patrias: Cuba y Puerto Rico. ${ }^{7}$

A Teruel no autorizaban visitas de corresponsales, pero sin que lo hubiera solicitado, López Sánchez fue citado por el Comité del Partido Comunista de España con el propósito de que fuera a esa ciudad a cumplir una misión secreta, ya que su cargo como corresponsal militar era la fachada perfecta y, de autorizarlo el mando de la división, -donde se desempañaba como redactor del periódico "Al Ataque"- no habría dificultades.

Al preguntar López Sánchez si podía hacerse acompañar de un corresponsal civil, miembro del Partido Comunista de Cuba, recibió su credencial y la autorización para hacerse acompañar por Carlos Montenegro, que estaba allí como corresponsal de la revista cubana "Mediodía." 
El día 24 de diciembre salieron de Madrid vía Valencia, con un chofer que no conocía bien los caminos para Teruel, lo que constituyó una pérdida de tiempo y finalmente el extravío de la ruta. Finalmente, en el cuartel de milicias, les indicaron la ruta a seguir para poder contactar al General Enrique Líster, pero les fue imposible llegar pues se libraba una batalla descomunal. Los tanques y la aviación nazi hacían incursiones cada vez más activas y potentes.

La noche del 30 de diciembre llegaron a Torrebaja donde estaba la División de Martínez Candón, y en la que había un cubano de apellido Cuervo. Finalmente López Sánchez y Montenegro pudieron hacer llegar el mensaje pero ya era demasiado tarde.

I niciaron el regreso y la última noche del año 1937 se encontraron en Albacete con la XV Brigada Internacional y el Batallón "Lincoln", del que era médico de ambulancias el capitán Luis Díaz Soto, médico cubano, uno de los más fraternos amigos de López Sánchez.

Aquella noche nevó tanto que el primer día de enero de 1938 los sorprendió sacando hielo de la entrada de la casa. Al mediodía continuaron para Cartagena, donde debían encontrar al capitán cubano J orge Agostini pero, como se atrasaron, ya había salido en misión para el frente.

El 3 de enero fueron al hospital de Murcia donde esperaban encontrar al médico cubano Eduardo Odio Pérez, pero les dijeron que el médico estaba en Madrid. Al día siguiente por la mañana, López Sánchez se presentó en las oficinas del Comité del Partido en Madrid, donde fue recibido por el entonces secretario general, Domingo Girón, a quien le informó los detalles y resultados de la misión. Recibió entonces la orden de salir de inmediato para Barcelona, entregándole un sobre con documentos del Comisario Pablo.

En Barcelona, López Sánchez contactó con Ramón Nicolau (Monguito), quien había llegado a la Ciudad Condal desde La Habana, acompañado por Emilio Laurent, Feliciano Maderne y Remberto O'Farrill, tres militares cubanos de experiencia cuya misión era asesorar a las tropas españolas en el manejo de las ametralladoras. Nicolau entregó a López Sánchez una carta de Blas Roca, quien le explicaba la urgencia de su regreso a Cuba debido al grave estado de salud de su padre.

En la comisión de cuadros se discutió sobre algunas incidencias del viaje a Teruel. En esa reunión, López Sánchez conoció a Bob Minor, miembro del Buró Político del Partido Comunista de EE. UU. Permaneció unos días más en Barcelona, tiempo que aprovechó para hablar por las ondas internacionales de la radio española, así como para establecer buenas relaciones con Neels Sbert, Ministro de Cultura de la Generalitat de Cataluña, quien había estado en Cuba y había conocido a Mella.

Su regreso había sido programado vía Nueva York; pero el día 31 de enero se produjo un bombardeo sobre Barcelona que destruyó una guardería infantil en la que perecieron decenas de niños y Nicolau y López Sánchez ayudaron a sacar los pequeños cuerpos mutilados de decenas de niños inocentes. Fue aquella su última tarea en España. A las doce de esa noche tomó el tren para la frontera.

Al siguiente día, temprano en la mañana, la aviación enemiga bombardeó la carretera, destruyendo la línea férrea, lo que obligó a evacuar rápidamente el tren con el equipaje y todos los pasajeros, la mayoría mujeres y niños. Se ordenó a los pasajeros avanzar por los costados de la línea férrea mientras esperaban otro tren que vendría a recogerlos. Los hombres debían dejar el equipaje, que sería después enviado a sus lugares de destino. López Sánchez llevaba dos maletas. En la más 
pesada llevaba documentos, fotos, libros y el sobre con las cosas de Pablo, entre las cuales estaba su certificación como miembro del Partido Comunista Español.

A las seis de la tarde los recogió otro tren y lograron pasar la frontera y continuar viaje a París. En la estación lo esperaban Félix Pita Rodríguez y Osmundo Illas. En París recibió instrucciones para ir a las oficinas del Partido Comunista de EE. UU., donde le entregaron el pasaje en barco para Nueva York.

Desde la Embajada de España en París, López Sánchez se comunicó por teléfono con Sbert y le narró lo sucedido, solicitándole el envío de su equipaje a París; pero cuando salió de allí la maleta no había llegado. Nunca la recibió.

En su libro dedicado a su entrañable amigo Pablo de la Torriente Brau, López Sánchez declara:

Uno de los acontecimientos que contribuyó decisivamente a mi formación como marxista y revolucionario - del cual vivo orgulloso- fue mi breve pero intensa participación en la guerra revolucionaria de España.

Han pasado ya algunas décadas pero se sigue recordando con amor y gratitud a las Brigadas Internacionales, porque están insertas en lo más noble de nuestra historia y la de otros países y como dijera Pasionaria- "en el corazón del pueblo español."77

\section{EL REGRESO A LA PATRI A}

A su regreso a Cuba, en 1938, López Sánchez siguió militando en el Partido Comunista, se graduó de doctor en medicina, con nota de Sobresaliente en el ejercicio de grado. A fines de ese año se le designó en el cargo de médico interno y de visita domiciliaria en el Centro Benéfico Jurídico de Trabajadores de Cuba, que dirigía su colega y compañero de la Brigada Internacional en la Guerra de España por la defensa de la República, doctor Luis Díaz Soto.

Desde 1942 se destacó en la organización y las luchas del gremio médico, perteneciendo al Comité Ejecutivo de la Federación Médica de Cuba primero y del Colegio Médico Nacional, después. Fue precisamente en este año en que comienza su interés por la historia de la medicina, constituyendo su primera obra la traducción al español del libro de Henry E. Sigerist, Socialized Medicine in the Soviet Union.

En 1943 ocupó la plaza de especialista en Dermatología en el Centro Benéfico Jurídico; en el Hospital de Infancia en 1946 y en el Hospital General Freyre de Andrade (Emergencias) en 1948.

Desde 1944 desempeñó innumerables actividades relacionadas con la Historia de la Medicina, entre las cuales se destaca su designación como Miembro correspondiente del Instituto Brazileiro de Historia de la Medicina y de la Sociedad de Historia de la Medicina de Venezuela, a lo que se añade su condición de Miembro fundador de la Sociedad Cubana de Historia de la Medicina y de la Sociedad Internacional de Historia de la Medicina. En 1945 fue Miembro fundador de la Sociedad Cubano Soviética de Ciencias Médicas y editor de su revista.

Unido a todo lo anterior, en las décadas de los años 1940 y 1950, estuvo una etapa en la clandestinidad y viajó en reiteradas oportunidades por América Latina, en 
misiones del partido. En esos avatares conoció en Chile a un médico socialista, Salvador Allende, a quien le unió una profunda amistad. ${ }^{8}$

\section{9, triunfo de la Revolución Cubana}

Al triunfo de la Revolución cubana se integró inmediatamente al proceso renovador, fue el médico personal del entonces Ministro de las Fuerzas Armadas y hoy Primer Secretario del Partido Comunista de Cuba y Presidente de los Consejos de Estado y de Ministros, Raúl Castro Ruz.

En tanto que Miembro de la Junta Directiva del Colegio Médico Nacional, explicó al Comandante Ernesto Guevara en su visita al mismo- la función de los comunistas dentro de esa organización gremial.

En 1960 ocupa el cargo de Asesor del Ministro de Salud Pública y en 1961, el cargo de Viceministro y Vicepresidente del Consejo Científico de dicho Ministerio. En este último año logra reabrir la Cátedra de Historia de la Medicina en la Escuela de Medicina de la Universidad de La Habana, y obtiene por concurso la plaza de Profesor de la disciplina. ${ }^{9}$ Fue el fundador del Museo de Historia de las Ciencias "Carlos J. Finlay" y su Director desde 1962 hasta 1974.

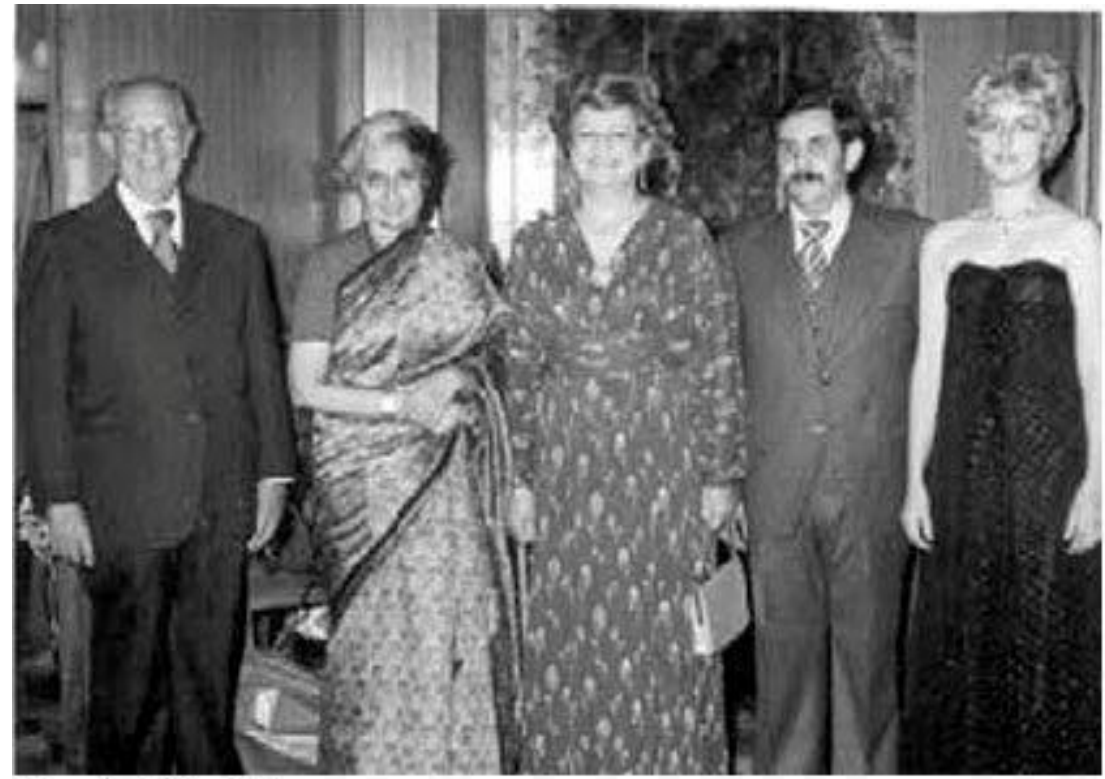

Fuente: Ob. cit.8.

Fig. 1. Junto a Indira Ghandi, siendo Embajador en la India (1974-1981).

En 1963 integró la Comisión Organizadora de la nueva Academia de Ciencias, de la que fue Vice-Presidente y Secretario, en el período 1964-1967. Desde 1968 hasta 1974 ocupó la plaza de Director del Instituto de Documentación Científico Técnica. En este último año resultó elegido Presidente de la Sociedad Cubana de Historia de la Medicina. En su primera intervención pública como tal, presentó la tesis titulada "Nueva significación de la historia de la medicina". En esta última responsabilidad estuvo apenas unos meses, pues en el propio año 1974 fue designado como Embajador de la República de Cuba en la India (Fig. 1), y además Embajador Concurrente en Pakistán, Bangla Desh, y en el Reino de Nepal por un período de 7 años. En ese tiempo conoció a la dirigente hindú, Indira Ghandi, a quien le unió una gran amistad (López Espinosa J A. Biografía del Profesor José López Sánchez. Premio Ciencias Sociales 1999). 
Al término de esa labor diplomática, en 1981, desempeñó el cargo de Director de Documentación del Ministerio de Relaciones Exteriores hasta 1984, en el que fue nombrado Embajador ante la Confederación Helvética (Suiza), cargo en el cual se mantuvo hasta 1989.

Desde su nuevo regreso a Cuba se dedica a la investigación en Historia de las Ciencias y la publicación de sus resultados en libros, artículos, monografías y otros. ${ }^{10}$

J osé López Sánchez fue un hombre de su tiempo, respondiendo en cada período de su vida a las exigencias del desarrollo de la sociedad a la que pertenecía.

Aunque su formación de base era la medicina, cuya especialización en Dermatología apenas pudo ejercer, su visión más social que biologista de la medicina le permitió compartir elementos de su vocación médica y sus inquietudes sociales, a sabiendas de que en no pocos casos los determinantes sociales del proceso salud-enfermedad son más decisivos que los propios componentes biológicos.

Su formación en la filosofía marxista-leninista le hizo interesarse tempranamente en la ciencia histórica, a sabiendas de que como dijera Marc Bloch- "la ignorancia del pasado no se limita a impedir el conocimiento del presente, sino que compromete, en el presente, la misma acción". ${ }^{11}$ En consecuencia, para un marxista como él, era obvia la necesidad de subsanar esta insuficiencia pues, en cumplimiento del llamado de Marx, de que "de lo que se trata no es de interpretar la realidad, sino de transformarla", ${ }^{12}$ solo la comprensión del presente le permitiría asumir las adecuadas acciones para transformar el futuro.

En el campo de la investigación histórica dedicó su mayor esfuerzo a dos grandes personalidades de las ciencias médicas cubanas, Tomás Romay Chacón y Carlos J. Finlay, cuyas obras impactaron de modo sobresaliente el desarrollo de la medicina en Cuba, no solo por su valor científico, sino especialmente por su valor social (Fig. 2).

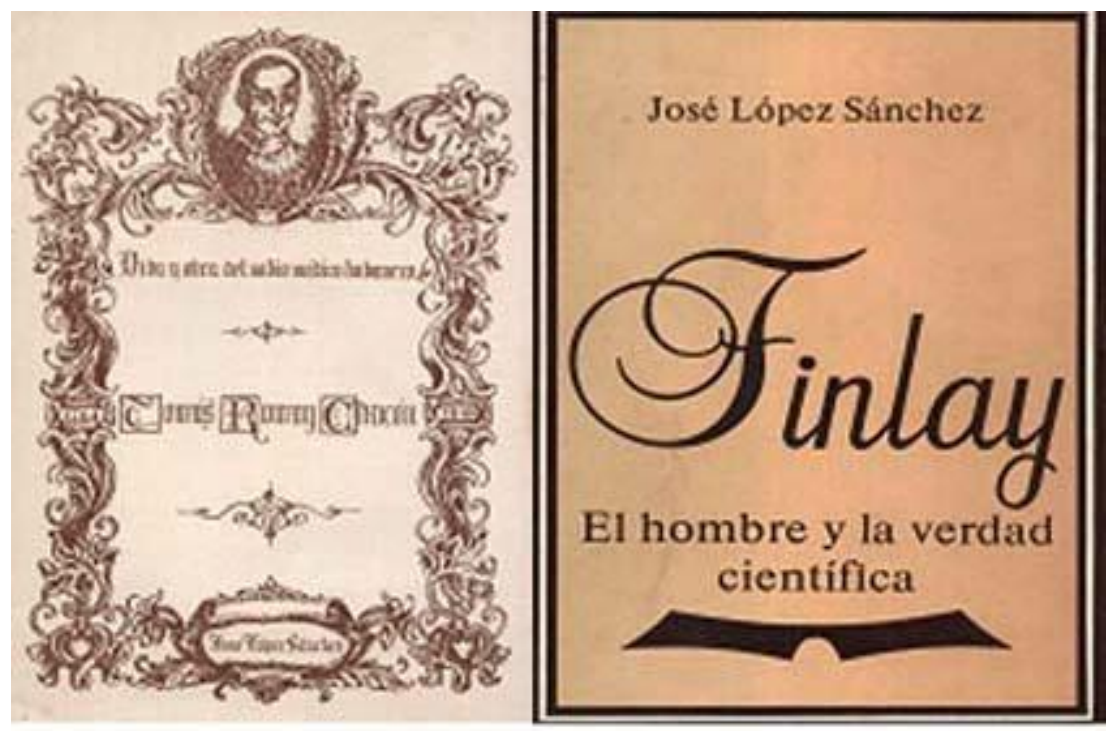

Fuente: Sociedad de Historia de las Ciencias.

Fig. 2. Sus dos grandes obras de Historia de la Medicina.

En el plano personal, López Sánchez demostró a lo largo de su vida su fidelidad a la causa revolucionaria, a su generación de los años 30s, a su Partido Comunista y a sus entrañables compañeros de lucha, especialmente a Pablo de la Torriente Brau, 
a quien dedicó una obra siendo ya nonagenario (Fig. 3). En ese libro, López Sánchez apenas dedica un par de cuartillas a relatar algunos hechos de su propia participación en la Brigada Internacional que luchara en defensa de la república española, sólo porque esos hechos personales están vinculados a la figura del héroe de Majadahonda. La imagen en la figura 3 fue captada por la autora en el momento de la presentación del libro en el Centro Cultural "Pablo de la Torriente Brau", en el 2003.

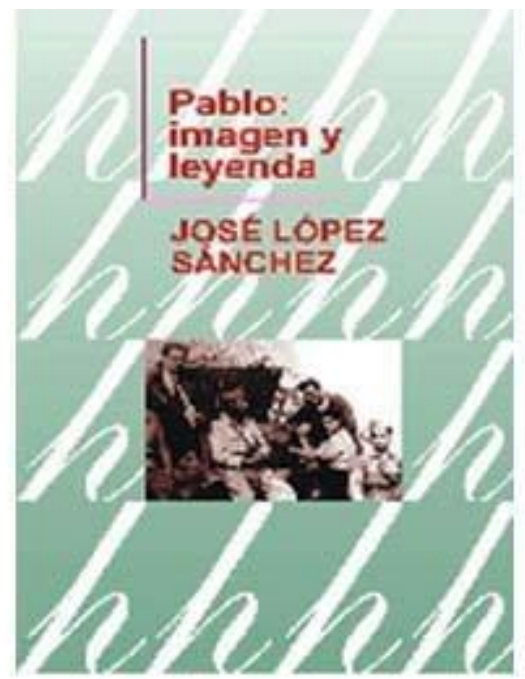

Fuente: foto tomada por la autora.

Fig. 3. Su testimonio de la Guerra Civil española, a través de la imagen en la memoria de su admirado amigo, Pablo de la Torriente Brau.

Su obra en el campo de la investigación en la Historia de las Ciencias fue reconocida al nombrársele Miembro de Honor de la Unión de Historiadores de Cuba y de la Sociedad Latinoamericana de Historia de la Ciencia y la Tecnología; Investigador de Mérito de la Academia de Ciencias de Cuba; Premio Nacional de Ciencias Sociales 1999 y la Orden de Mérito Científico "Carlos J. Finlay". En tanto que la Universidad le reconoció otorgándole la condición de Profesor de Mérito del entonces Instituto Superior de Ciencias Médicas de La Habana, poco tiempo antes de su fallecimiento.

¡Honrar, honra! -decía J osé Martí- y la autora también quiere honrar la memoria del Profesor J osé López Sánchez, en el centenario de su natalicio, con el modesto homenaje que contiene este breve artículo.

\section{REFERENCI AS BI BLI OGRÁFICAS}

1. Cairo A. Mella 100 años. Selección, prefacio y nota. Santiago de Cuba: Editorial Oriente; 2003.

2. de la Torriente Brau P. Hombres de la Revolución. Páginas escogidas. La Habana: Impresora Universitaria Andrés Voisin; 1973.

3. Depestre Catony L. Cuba en citas. 1899-1952. La Habana: Editorial Gente Nueva; 1987. 
4. Roa García R. Retorno a la alborada. Universidad Central de las Villas: Dirección de Publicaciones Universitarias; 1964.

5. ___._. La revolución del 30 se fue a bolina. La Habana: Instituto del Libro, Ediciones Huracán; 1969.

6. de la Torriente Brau P. ¡Arriba muchachos! La Habana: Ediciones La Memoria; 2001.

7. López Sánchez J. Pablo: Imagen y Leyenda. La Habana: Ediciones La Memoria; 2003.

8. Beldarraín Chaple E. José López Sánchez (1911-2004) en el panorama científico cubano. Bol Mex His Fil Med. 2010; 13(1): 16-20.

9. Delgado García G. El doctor J osé López Sánchez (1911-2004), historiador de la medicina cubana. Cuad Hist Salud Pública No. 99. La Habana: Editorial Ciencias Medicas; 2004.

10. López Espinosa J A. Obituario Profesor J osé López Sánchez. ACIMED [Internet]. 2004 [citado 23 Mar 2012]; 12(5). Disponible en:

http://bvs.sld.cu/revistas/aci/vol12_5_04/aci13504.htm

11. Bloch M. Introducción a la Historia. México-Buenos Aires: Fondo Cultura Económica; 1964.

12. Marx K, Engels F. Obras Escogidas. Moscú: Editorial Progreso; 1973.

Recibido: 13 de junio de 2011.

Aprobado: 19 de octubre de 2011.

María del Carmen Amaro Cano. Facultad de Ciencias Médicas "General Calixto García". Calle 27 y G. El Vedado 10400. La Habana, Cuba. Correo electrónico: amaro@infomed.sld.cu 